\title{
Failure of a water-bath design intravenous fluid warmer
}

\author{
Paul Adrian Clarke, FRCA · Malcolm J. Thornton, FRCA
}

Received: 4 June 2009/Accepted: 22 July 2009/Published online: 21 August 2009

(C) Canadian Anesthesiologists' Society 2009

\section{To the Editor,}

We write to highlight the failure of an intravenous fluid warming device, HOTLINE ${ }^{\circledR}$ HL-290 Blood and Fluid Warmer (Smiths Medical International, Kent, UK), which could have resulted in harm to the patient. Fluid warmer failures are becoming more prevalent, as evidenced by the United Kingdom Medicines and Healthcare Products Regulatory Agency (MHRA) issuing a recall of all Level 1 SIMS fluid warmer sets. ${ }^{1,2}$ The fluid warmer in our case is designed differently. Warm water is pumped into a counter-current system around an inner sterile infusion lumen. The warming fluid is kept in a non-sterile water bath, emphasizing the need for extreme care with all water-bath fluid warmers. ${ }^{3}$

Our patient received general anesthesia for laparoscopic radical prostatectomy. Apparently, the warming infusion tubing had been tested prior to surgery. However, after $2 \mathrm{~L}$ of intravenous fluid had been connected and pressurized, the water bath overflowed. A leak within the coaxial infusion tubing, which allowed mixing of the sterile intravenous fluid and the non-sterile warming fluid, was immediately suspected. The infusion was stopped immediately, and the administration set was disconnected from the patient. An entirely new set was assembled, tested for leaks, and connected, and the device functioned uneventfully for the remainder of the procedure. The patient's serum electrolyte values remained within normal limits, and the blood cultures were devoid of microbiological growth. Fortunately, the patient completely recovered postoperatively with no signs of sepsis or dilutional electrolyte imbalance. The warming fluid culture revealed heavy growth of pseudomonas and

P. A. Clarke, FRCA $(\square) \cdot$ M. J. Thornton, FRCA Royal United Bath NHS Trust, Bath, Somerset, UK e-mail: paclarke@doctors.org.uk coliform bacteria. Furthermore, cultures from another three water-bath warmers, which were in the theatre suite at the same time, grew diptheroid and fungal species.

On subsequent examination of the infusion tubing, a hairline circumferential crack was visible within the wall of the inside lumen. When the water-bath warmer was re-tested, fluid flowed from the outside warming lumen to the inside sterile lumen. We considered this problem to be a manufacturing defect since no direct trauma to the device was observed and the external wall was undamaged. The faulty equipment was returned to the manufacturer for further investigation, and the matter was reported to the MHRA.

The manufacturers recommend a number of options for maintaining warming fluid sterility ${ }^{4}$, specifically, if either sterile water or $35 \%$ isopropyl alcohol in distilled water is used as the warming fluid, the fluid should be changed and a disinfection protocol followed every 30 days. The alternative is to use a $0.3 \%$ hydrogen peroxide solution, which requires the solution to be replaced and the water bath disinfected every 12 months. We use sterile water in our institution, and each hotline is discarded after one use to ensure frequent turnover of the warming fluid.

There are important patient safety issues related to this case. It is impossible to know how much of the grossly hypotonic, non-sterile warming fluid entered the patient's circulation, although the lack of detectable hemolysis, stability of the serum electrolyte measurements, and lack of sepsis postoperatively suggest the amount, if any, was small. Professionals who use this fluid warmer should be alerted to the possibility that warming fluid and intravenous fluid could be mixed. This risk can be eliminated by using the electric hotplate design of fluid warmer, because there is no chance of fluid contamination. If any fluid is observed around the outside of the tubing, then a leak must have occurred. 
Competing interests None declared.

\section{References}

1. MDA/2007/064 —Fluid warming set-Level 1 normothermic IV fluid administration and irrigation sets-D/DI and IR/IRI series manufactured by Smiths Medical. Available from URL: MHRA website, http://www.mhra.gov.uk/Publications/Safetywarnings/Medical
DeviceAlerts/CON2032043, issued 15 August 2007 (accessed November 2008)

2. Wilson $S$, Szerb J. Failure of an iv fluid warming device. Can J Anesth 2007; 54: 324-5.

3. Anonymous. Hazard report. Greater vigilance urged in use of SIMS Level 1 Hotline fluid warmers to detect leaks in warming set. Health Devices 2000; 29: 478-80.

4. Smiths Medical. HOTLINE blood and fluid warmer HL-90 operator's manual, 2005. 